\title{
The direct deleterious effect of Th17 cells in the nervous system compartment in multiple sclerosis and experimental autoimmune encephalomyelitis: one possible link between neuroinflammation and neurodegeneration
}

\author{
Rodica Balasa ${ }^{1}$, Smaranda Maier ${ }^{1 *}$, Laura Barcutean ${ }^{1}$, Adina Stoian², \\ Anca Motataianu ${ }^{1}$ \\ 1. Department of Neurology, George Emil Palade University of Medicine, Pharmacy, Science, \\ and Technology of Targu Mures, Romania \\ 2. Department of Pathophysiology, George Emil Palade University of Medicine, Pharmacy, Science, \\ and Technology of Targu Mures, Romania
}

\begin{abstract}
The processes of demyelination and neurodegeneration in the central nervous system (CNS) of multiple sclerosis (MS) patients and experimental autoimmune encephalomyelitis (EAE) are secondary to numerous pathophysiological mechanisms. One of the main cellular players is the Th17 lymphocyte. One of the major functions described for Th17 cells is the upregulation of pro-inflammatory cytokines, such as IL-17 at the level of peripheral and CNS inflammation. This review will focus on the newly described and unexpected, direct role played by the Th17 cells in the CNS of MS patients and EAE models. Th17 and their main cytokine, IL-17, are actively involved in the onset and maintenance of the immune cascade in the CNS compartment as Th17 were found to achieve brain-homing potential. Direct interaction of myelin oligodendrocyte glycoprotein - specific Th17 with the neuronal cells firstly induces demyelination and secondly, extensive axonal damage. The Th17 cells promote an inflammatory B cell response beyond the BBB through the presence of infiltrating Th follicles. Due to their role in preventing remyelination and direct neurotoxic effect, Th17 cells might stand for an important connection between neuroinflammation and neurodegeneration in a devastating disease like MS. The Th17 cell populations have different mechanisms of provoking an autoimmune attack not only in the periphery but also in the CNS of MS patients.
\end{abstract}

Keywords: multiple sclerosis, Thelper 17, blood brain barrier, ectopic lymphoid structure NG2+ Received: $3^{\text {rd }}$ December 2019; Accepted: $2^{\text {nd }}$ January 2020; Published: $9^{\text {th }}$ January 2020

*Corresponding author: Smaranda Maier, Department of Neurology, George Emil Palade University of Medicine, Pharmacy, Science, and Technology of Targu Mures, Romania. E-mail: maier_smaranda@yahoo.com 


\section{Introduction}

Multiple sclerosis (MS), an autoimmune and neurodegenerative disease of the central nervous system (CNS) is caused partially by a genetic polymorphism and various environmental factors (1).

A major component of the immunopathology of MS is the T cell-driven inflammatory attack from the peripheral immune system towards the CNS (2). MS inflammatory demyelination is buckled with neurodegeneration, albeit the primary mechanism underlying this phenomenon remains in debate.

T helper (Th) 17 cells were described in 2005 by Harrington et al (3). Ever since, various clinical and experimental research studies have attributed numerous roles for this group of Th cells in the immunopathology of MS and experimental autoimmune encephalomyelitis (EAE). The Th17 cells are key players in MS pathogenesis during all the disease phases. Until recently, the medical literature has attributed to the Th17 cells the delineation of initiation and maintenance of the immune attack in MS and EAE. The scientific works regarding the two most important aspects of the implication of Th17 cells in MS immunopathogenesis are chronologically as follows: in 2009, Durelli et al. reported that Th17 lymphocytes increase and expand in the peripheral blood of patients with active MS; in 2013, Rostami et al. demonstrated that the blood-brain barrier (BBB) can be efficiently crossed by the Th17 cells using different pathways from Th1 cells, thus promoting its disruption. The BBB breakthrough is a complex process, sustained by the involvement of Th17 cells. The possibility of Th17 cells to induce the activation of other inflammatory cells in the CNS, to maintain the immune process inside the CNS and to inhibit the remyelination of demyelinated axons was recently described (4-8). Undoubtedly, Th17 cells contribute both to the onset and to the progression of immunological mechanisms in MS/EAE. Th17 lymphocytes are involved in the self-entertainment of the brain inflammation in MS patients with sustained myelin and axonal damage (8-10).

In EAE, the presence of Th17 cells into the parenchymal CNS white matter that mediates CNS inflammation is possible according to a two-wave proposed hypothesis. First wave is represented by the cell traffic through the choroid plexus into the subarachnoid space, where the Th17 cells encounter antigen presenting macrophages (meningeal antigen presenting cells); the second wave is formed by these re-stimulated Th17 cells that undergo clonal expansion and migrate across the glia limitans basement membrane. Once they reach the white matter, the Th17 cells will initiate tissular destruction, demyelination and axonal injury, that will subsequently lead to neurodegeneration (11-13).

The present review will focus on the recently described and unexpected role of the Th17 cells in the CNS of MS and EAE. These cells and their main secreted cytokine, IL-17 are actively involved in the maintenance of the immune cascade in the CNS compartment as Th17 cells were found to achieve brain-homing potential (11). The levels of Th17 lymphocytes are elevated in the cerebrospinal fluid (CSF) and in the perivascular tissue of clinically isolated syndrome or MS patients during the relapses, and respectively, in the demyelinating brain lesions $(11,14,15)$.

\section{Th17 cell induce direct neuronal dysfunction}

The Th17 cells attack neurons during the acute phase of EAE through the formation of synapse-like contacts with neurons. Kebir et al. described a prevalence of Th17-mediated attacks in MS types with main cerebral involvement unlike spinal forms of MS, where Th1 lymphocytes are predominant (8). Siffrin et al. found direct 
interactions between Th17 cells and neuronal cells at the level of the brainstem, mainly during the EAE peak. The direct interaction between myelin oligodendrocyte glycoprotein - specific Th17 with the neuronal cells induces demyelinating lesions and secondary extensive axonal damage. The Th17 cells induce fluctuations in the neuronal intracellular $\mathrm{Ca}^{2+}$ concentration, marking the initial neuronal damage. In neuroinflammatory lesions, the major phenomenon found in the early neuronal damage processes was excitotoxicity (Figure1). Numerous different pathophysiologic conditions (traumatic brain injury, stroke, autoimmune diseases) share the same pathways that lead to neurodegeneration through the sustained influx of $\mathrm{Ca}_{2}{ }^{+}$that induces Wallerian degeneration and protease activation that cause cytoskeletal and neurofilament degradation. Th17 cells exhibit a preferential ability to engage in sustained contacts with neurons (16).
In EAE, the neuron/Th17 cell interactions lead to localized $\mathrm{Ca}^{+}$oscillations provoking axonal and perikaryonal injuries. These injuries are reversible to the administration of the N-methyl-D-aspartate receptor antagonist MK801. This finding demonstrates that Th17 cells have an important, pivotal role in neuronal loss, and not only in demyelination. These data might be the missing piece of the puzzle in immune-mediated neurodegeneration. There is a very complex immune-neuronal dynamics during demyelinating pathologies like MS or $\operatorname{EAE}(8,17,18)$.

\section{Th17 cells and ectopic lymphoid-like structures}

In 2007, Magliozzi et al. found that clustered populations of infiltrating immune cells can be found in the brain of about $40 \%$ of the secondary progressive MS patients, especially in the

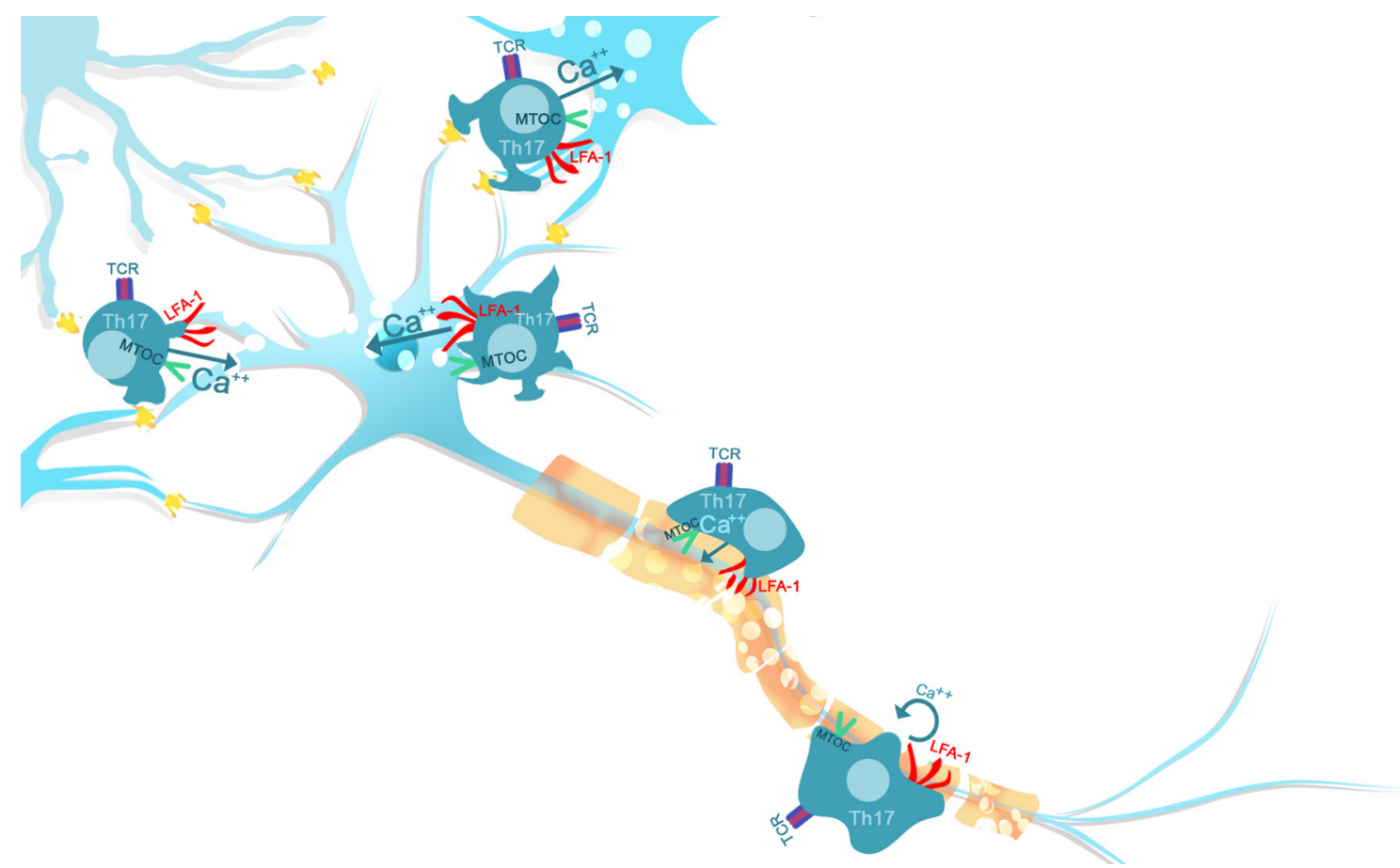

Fig. 1. Direct Th17 cell attack on neurons (MTOC: microtubule organizing center; TCR: T-cell receptor; LFA-1: Lymphocyte function-associated antigen 1; Th17: T helper 17 lymphocyte) 
meningeal regions, forming lymphocyte-rich aggregates (19). These aggregates stimulate the cortical demyelination and axonal atrophy that are associated with the progression of neurological dysfunction in MS cases. Ectopic lymphoid structures (ELS) are found in the brain regions with numerous cortical demyelinating lesions (20). These 'follicles' resemble the follicles found in spleen or tonsils, known as secondary lymphoid tissues (19). Th17 cells that produce IL-17 play important roles in the development of these ELS as was demonstrated in animal models of chronic demyelination. Inside these follicles, in the presence of IL-21, B cells differentiate, they promote inflammation and maintain an autoreactive $\mathrm{T}$ and $\mathrm{B}$ cell population beyond the BBB. This microenvironment of 'germinal centres' supports and balances the interactions of B cells with follicular Th cells. A number of experimental studies performed on mice that express more IL-17, showed that blocking IL-17 signalling decreased the interactions between $\mathrm{B}$ cells and $\mathrm{CD}^{+}$cells needed for the formation of ELS $(21,22)$. A research performed on transferred Th17 cells showed that these cells begin to take a Th follicular-like phenotype in the mice brain during EAE and also, when transferred into healthy mice recipients, spontaneous ELS formation was obtained (23). IL-17 and IL-21 are important for the formation of spontaneous ELS but also for the development of pathogenic autoantibodies in the CNS. The mechanism through which IL-17 is involved in this process is the following: IL17 upregulates signalling proteins that promote the capacity of follicular Th cells to form conjugates with B cells in the light zone of germinal centres in CNS and promotes follicular Th cells differentiation and stabilisation $(21,22,24)$.

Furthermore, the germinal centres include both follicular $\mathrm{T}$ cells and follicular regulatory $\mathrm{T}$ cells that maintain an optimal response in preventing the auto-emergence of the autoreactive B cells
(25, 26). In MS patients, follicular regulatory cells appear supressed compared to healthy controls, most likely secondary to a proinflammatory phenotype of circulating Th cells or due to a deficit in CTLA-4 signalling, which is essential for the follicular regulatory Th cell function. Dhaeze $\mathrm{T}$ et al, (2015) reports that the ratio between follicular $\mathrm{T}$ cells and follicular regulatory $\mathrm{T}$ cells is increased in patients compared to controls, indirectly translated as a deficit of pro-inflammatory Th suppression $(27,28)$.

There is a close relationship between B cells and Th17 cells, leading to an elevated number of B cells and ELS as a mark for Th17 signature in MS $(22,24)$. Th17 cells promote an inflammatory B cell response inside the CNS through the presence of infiltrating Th follicles (29). Likewise, B cells can support the survival and activities of Th17 cells in MS. In vitro assays showed that the B cells from MS patients display the capacity of coordinating Th17 riposte against neuro-antigens (30). As a result, in 2018 Quinn and Axtell described an 'inflammatory axis' consisting of Th17 cells, B cells and follicular Th cells (31). Th17 cells through their implication in the formation of the subpial follicles, have a contributory role in the underlying subpial grey matter pathology (4). The propagation of neuroinflammation inside the CNS compartment is done by Th17 cells through the interaction between the lymphotoxin $\alpha \beta(\mathrm{LT} \alpha \beta)$, a ligand situated on Th17 cells and the receptor LT $\beta$ R situated on stromal cells. The Th17 cells promote the meningeal microenvironmental re-modelation that has a deleterious effect in MS evolution by inducing the production of proinflammatory cytokines, chemokines and various extracellular matrix proteins. There are speculations that the circulation of these LT $\alpha \beta$-Th 17 cells through the CSF might remodel the meningeal stroma in MS patients and increase the severity of the disease even in the early cases of MS (32) (Figure 2). 


\section{Th17 and IL-17 inhibits remyelination through downregulation of $\mathrm{NG2}^{+}$cells}

Since the description of MS made by Charcot, demyelination is the hallmark of this disease (33, 34). Oligodendrocyte progenitor cells (OPC) are able to differentiate into myelinating oligodendrocytes but also to proliferate and migrate in the demyelinated regions of the CNS. Neuroplasticity is a rather general and blurry term but it is certain that one constituent mechanism is the capacity of OPC to proliferate, to advance to the demyelinated region and to induce the genesis of new myelinating oligodendrocytes. Over the course of MS, OPC tend to gradually lose their remyelination capacity. One of the responsible phenomena is represented by the difficulty of the OPC to be "activated." Initially, remyelination often follows demyelination. In order for this process to take place, newly recruited OPCs secrete new myelin and restore the myelin sheath around the axons, protecting them from neurodegeneration. Unfortunately, after a variable period of time, the remyelinating process is exceeded by the speed of demyelination that creates a milieu that limits the efficacy of the reparatory process (35).

The OPCs express gangliosides that are recognized by the neuron-glial antigen2 (NG2, a surface proteoglycan) and the receptor of platelet-derived growth factor alpha. The maturation and the proliferation of NG2 cells generate the remyelination process. NG2 cells are extensively dispersed throughout the CNS. NG2 are a distinct cell population residing inside the CNS. They are distinct from neuronal cells, oligodendrocytes and astrocytes and close to OPCs, and during development they can generate either oligodendrocytes or astrocytes (35).

$\mathrm{NG}^{+}$glial cells are a specific macroglia population in CNS. The proliferation and maturation of $\mathrm{NG}^{+}$cells are at the base of the remyelina-

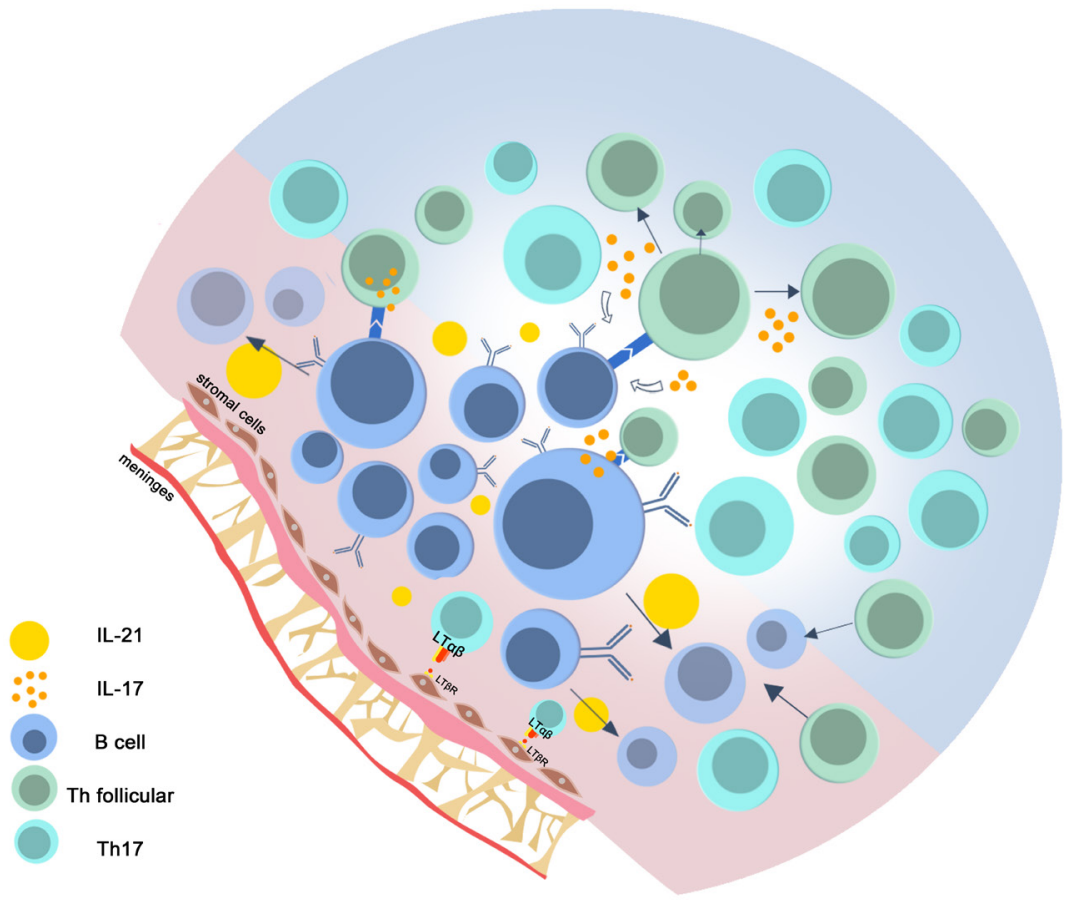

Fig. 2. Involvement of Th17 together with B cells and Th follicular in the "inflammatory axis" of the CNS 
tion process. Following demyelination, $\mathrm{NG}^{+}$ glia proliferates and expresses NG2 that furthermore contributes the to myelin repair indirectly by giving rise to the oligodendrocytes but also directly by resolution of the glial scar $(36,37)$.

IL-17 participates in the pathogenesis and development of EAE by contributing to brain destruction through demyelination and neurodegeneration (axonal injury). IL-17-mediated proinflammatory effects are supplemented by the role of IL-17 in preventing the remyelination processes of demyelinated axons. It was stated that IL-17 also influences the remyelinating processes, by stimulating oligodendrocyte apoptosis. From this point of view, in EAE, one of the main CNS cellular targets of IL-17 is $\mathrm{NG}^{+}$glia. Act1, an adaptor for IL-17 receptors also known as nuclear factor kappa-light-chain-enhancer of activated $B$ cells (NF- $\kappa$ B activator 1$)$ is necessary for IL-17-mediated inflammatory responses. Kang et al. showed that in experimental murine models, IL-17 induced in $\mathrm{NG}^{+}$glial cells typical inflammatory mediated expressions through
Act1. A reduced EAE severity was experimentally obtained by the deletion of Act 1 from $\mathrm{NG}^{+}$ glia $(35,37)$ (Figure 3$)$. The results published by Zhang et al, determined that in forms of EAE that are IL-17-mediated, $\mathrm{NG}^{+}$glia might be a CNS cellular target (38). Secondary to neuroinflammation, many studies have found morphological transformation of $\mathrm{NG}^{+}$glia $(39,40)$. During the crosstalk between oligodendrocyte, astrocyte and microglia throughout demyelination and remyelination in MS brain, it seems that the Th17 cells play an important role towards neurodegeneration (41).

IL-17 also displays crucial inhibitory effects on the in vitro development of oligodendrocyte lineage cells by reducing their survivability through the stimulation of the tumour necrosis factor (TNF)- $\alpha$-mediated cell death cascade that furthermore induces oligodendrocyte loss through apoptosis. Active oligodendrocytes substantially contribute to the repair processes in demyelinated CNS demyelinated lesions. Lately, there has been increased evidence that one important play-

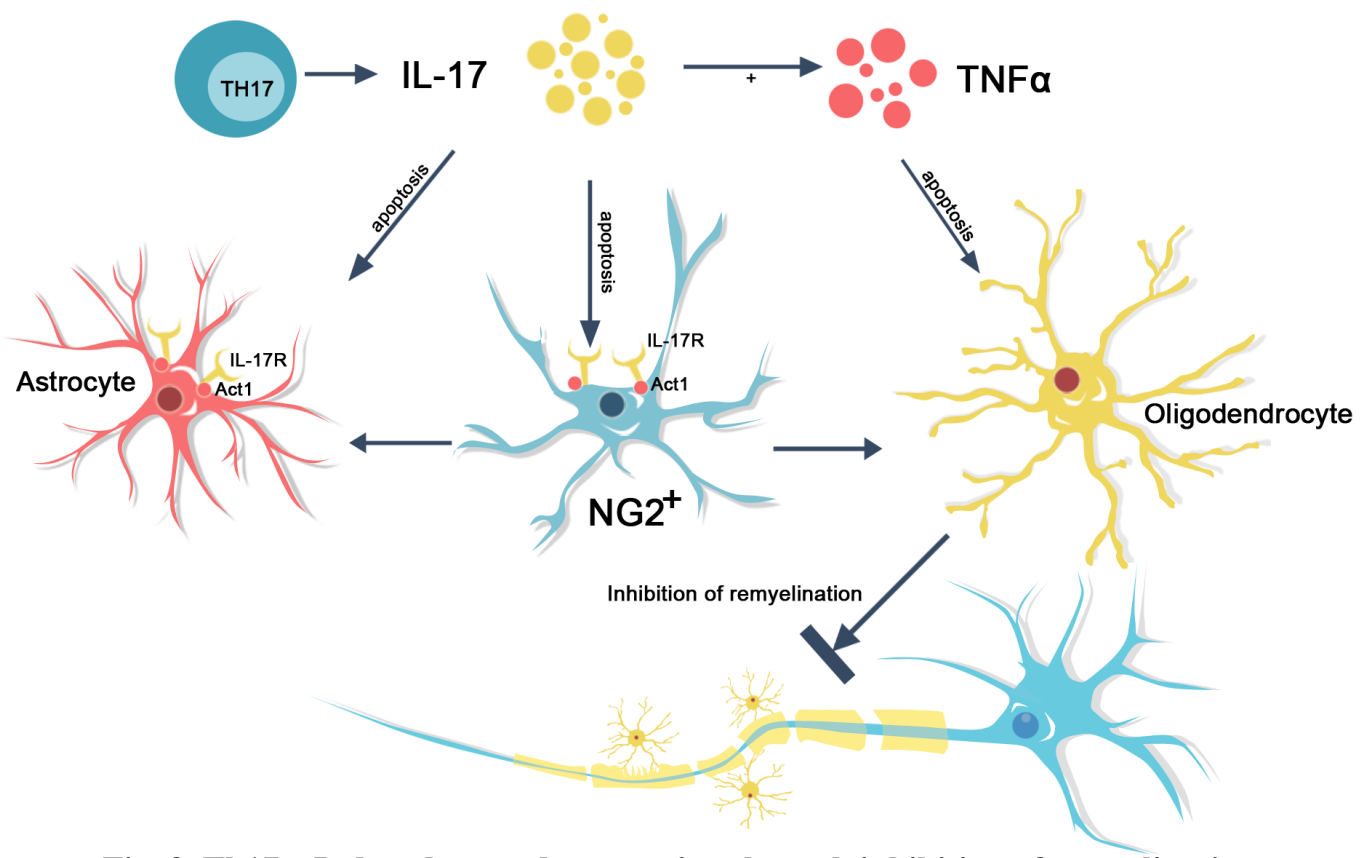

Fig. 3. Th17 - Induced neurodegeneration through inhibition of remyelination 
er in the vascular pathology in MS might be the IL-17R, receptor located on astrocytes and microglia, therefore IL-17 can also interact directly with these cells (9). This might represent the one link between inflammation and neurodegeneration in MS and also the explanation for the heterogenous progressive neurological deficits (42). Of course, the essential question remains open: is oligodendrocyte injury in MS the cause or the consequence of the inflammation? (Figure 3)

\section{Conclusions}

In the immunopathology of MS/EAE, Th17 cells are involved early carrying deleterious effects on two very important compartments: the peripheral immune compartment that is activated towards a proinflammatory status and the $\mathrm{BBB}$, which is profoundly disrupted. In the central nervous compartment, Th17 cells continue to have a self-maintained proinflammatory (but also a neurodegenerative) role. Direct interaction of myelin oligodendrocyte glycoprotein - specific Th17 with neuronal cells creates demyelinating lesions and secondary extensive axonal damage. The development of ELS found in the brain of the early but severe cases of MS is supported by interleukins that are secreted by the Th17 cells, such as IL-17 and IL-21 and is a cause of local CNS demyelination. Another effect of the Th17 cells/IL-17 beyond the BBB is the interference with the remyelination process by inhibiting the oligodendrocytes through different mechanisms. Due to their role in preventing remyelination and direct neurotoxic effect, Th17cells might stand for an important connection between neuroinflammation and neurodegeneration in a devastating disease such as MS. Some of the immune-mediated mechanisms presented might be therapeutically accessible in order to prevent dysfunction in the central nervous compartment.

\author{
Abbreviations \\ Act1 - IL-17R adaptor \\ BBB - blood-brain barrier \\ CNS - central nervous system \\ CSF - cerebrospinal fluid \\ EAE - experimental autoimmune encephalomy- \\ elitis \\ ELS - ectopic lymphoid structures \\ LT $\alpha \beta$ - lymphotoxin $\alpha \beta$ \\ LT $\beta R$ - lymphotoxin $\beta$ receptor \\ MS - multiple sclerosis \\ $\mathrm{NF}-\kappa \mathrm{B}$ activator 1 - kappa-light-chain-enhancer \\ of activated $\mathrm{B}$ cells \\ $\mathrm{NG} 2+$ - neuron-glial antigen2+ \\ NMDA - N-methyl-D-aspartate \\ OPC - oligodendrocyte progenitor cells \\ $\mathrm{Th}-\mathrm{T}$ helper
}

\section{Acknowledgement}

This review article was supported by 2017 Merck Grant for Eastern European Countries (No 548/2018). The authors wish to thank their co-author, BL, for the figure design and the conceptualization of the images, which may be used upon request from a third party).

\section{Authors contribution}

BR (Conceptualization, Methodology, Writing original draft preparation, Funding Acquisition) BL (Investigation, Resources, Software, Conceptualization of the figures and drawings)

MS (Investigation, Resources, Visualisation)

SA (Investigation, Formal Analysis)

MA (Conceptualization, Writing - review and editing, Supervision) 


\section{Conflict of interest}

The authors declare no conflict of interest.

\section{References}

1. Cakina S, Ocak O, Ozkan A, Yucel S, Karaman HIO. Vitamin D receptor gene polymorphisms in multiple sclerosis disease: A case-control study. Rev Romana Med Lab. 2018;26(4):489-95. DOI: 10.2478/rrlm2018-0028

2. Arellano G, Acu-a E, Reyes LI, Ottum PA, De Sarno P, Villarroel L, et al. Th1 and Th17 Cells and Associated Cytokines Discriminate among Clinically Isolated Syndrome and Multiple Sclerosis Phenotypes. Front Immunol. 2017 Jun 30; 8:753. DOI: 10.3389/fimmu.2017.00753

3. Harrington LE, Hatton RD, Mangan PR, Turner H, Murphy TL, Murphy KM, et al. Interleukin 17-producing CD4+ effector T cells develop via a lineage distinct from theT helper type 1 and 2 lineages. Nat Immunol. 2005 Nov;6(1):1123-32. DOI: 10.1038/ni1254

4. Durelli L, Conti L, Clerico M, Boselli D, Contessa G, Ripellino P, et al. T-helper 17 cells expand in multiple sclerosis and are inhibited by interferon-beta. Ann Neurol. 2009; 65:499-509. DOI: 10.1002/ana.21652

5. Rostami A, Ciric B. Role of Th17 cells in the pathogenesis of CNS inflammatory demyelination. J Neurol Sci. 2013 Oct; 333:76-87. DOI: 10.1016/j.jns.2013.03.002

6. Brucklacher-Waldert V, Stuerner K, Kolster M, Wolthausen J, Tolosa E. Phenotypical and functional characterization of T helper 17 cells in multiple sclerosis. Brain. 2009 Dec;132:3329-41. DOI: 10.1093/brain/ awp289

7. Jadidi-Niaragh F, Mirshafiey A. Th17 cell, the new player of neuroinflammatory process in multiple sclerosis. Scand J Immunol. 2011 Jul;74:1-13. DOI: 10.1111/j.1365-3083.2011.02536.x

8. Kebir H, Kreymborg K, Ifergan I, Dodelet-Devillers A, Cayrol R, Bernard M, et al. Human TH17 lymphocytes promote blood-brain barrier disruption and central nervous system inflammation. Nat Med. 2007 Oct;13:1173-5. DOI: 10.1038/nm1651

9. Pelletier M, Maggi L, Micheletti A, Lazzeri E, Tamassia N, Costantini C, et al. Evidence for a crosstalk between human neutrophils and Th17 cells. Blood 2010 Jan; 115 (2):335-43. DOI: 10.1182/blood-2009-04-216085

10. Kostic M, Stojanovic I, Marjanovic G, Zivkovic N, Cvetanovic A. Deleterious versus protective autoimmunity in multiple sclerosis. Cell Immunol. 2015; 296:122-32. DOI: 10.1016/j.cellimm.2015.04.006

11. Chihara N. Dysregulated T cells in multiple sclerosis. Clin Exp Neuroimmunol. 2018; 9:20-29. DOI: 10.1111/ cen3.12438

12. Reboldi A, Coisne C, Baumjohann D, Benvenuto F, Bottinelli D, Lira S, et al. C-C chemokine receptor 6-regulated entry of TH-17 cells into the CNS through the choroid plexus is required for the initiation of EAE. Nat Immunol. 2009 May;10:514-23. DOI: 10.1038/ ni. 1716

13. Lutz SE, Smith JR, Kim DH, Olson CVL, Ellefsen $\mathrm{K}$, Bates JM, et al. Caveolin1 is required for Th1 cell infiltration, but not tight junction remodeling, at the blood-brain barrier in autoimmune neuroinflammation. Cell Rep. 2017 Nov;21:2104-2117. DOI: 10.1016/j.celrep.2017.10.094

14. Tahmasebinia F, Pourgholaminejad A. The role of Th17 cells in auto-inflammatory neurological disorders. Prog Neuropsychopharmacol Biol Psychiatry. 2017 Oct;79:408-416. DOI: 10.1016/j.pnpbp.2017.07.023

15. Wojkowska DW, Szpakowski P, Ksiazek-Winiarek D, Leszczynski M, Glabinski A. Interactions between neutrophils, Th17 cells, and chemokines during the initiation of experimental model of multiple sclerosis. Mediators Inflamm. 2014;2014:590409. DOI: 10.1155/2014/590409

16. Siffrin V, Radbruch H, Glumm R, Niesner R, Paterka $\mathrm{M}$, Herz $\mathrm{J}$, et al. In vivo imaging of partially reversible th17 cell-induced neuronal dysfunction in the course of encephalomyelitis. Immunity. 2010 Sep;33(3):424-36. DOI: 10.1016/j.immuni.2010.08.018

17. Haqqani AS, Stanimirovic DB. Intercellular interactomics of human brain endothelial cells and Th17 lymphocytes: a novel strategy for identifying therapeutic targets of CNS inflammation.Cardiovasc Psychiatry Neurol. 2011;2011:175364. DOI: 10.1155/2011/175364

18. Liblau RS, Gonzalez-Dunia D, Wiendl H, Zipp F. Neurons as targets for $\mathrm{T}$ cells in the nervous system. Trends Neurosci. 2013; 36(6):315-24. DOI: 10.1016/j. tins.2013.01.008

19. Magliozzi R, Howell O, Vora A, Serafini B, Nicholas R, Puopolo M, et al. Meningeal B-cell follicles in secondary progressive multiple sclerosis associate with early onset of disease and severe cortical pathology. Brain. 2007 Apr;130:1089-104. DOI: 10.1093/brain/awm038

20. Howell OW, Reeves CA, Nicholas R, Carassiti D, Radotra B, Gentleman SM, et al. Meningeal inflammation is widespread and linked to cortical pathology in multiple sclerosis. Brain. 2011 Sep;134:2755-71. DOI: 10.1093/brain/awr182

21. Hsu HC, Yang P, Wang J, Wu Q, Myers R, Chen J, et al. Interleukin 17-producing $\mathrm{T}$ helper cells and interleukin 17 orchestrate autoreactive germinal center development in autoimmune BXD2 mice. Nat Immunol. 2008 Feb;9:166-75. DOI: 10.1038/ni1552

22. Ding Y, Li J, Wu Q, Yang P, Luo B, Xie S, et al. IL17RA is essential for optimal localization of follicular Th cells in the germinal center light zone to promote autoantibody-producing B cells. J Immunol. 2013 Aug;191:1614-24. DOI: 10.4049/jimmunol.1300479

23. Peters A, Pitcher LA, Sullivan JM, Mitsdoerffer M, 
Acton SE, Franz B, et al. Th17 cells induce ectopic lymphoid follicles in central nervous system tissue inflammation. Immunity 2011 Dec;35(6):986-96. DOI: 10.1016/j.immuni.2011.10.015

24. Wichner K, Stauss D, Kampfrath B, Krüger K, Müller $\mathrm{G}$, Rehm A, et al. Dysregulated development of IL17- and IL-21-expressing follicular helper T cells and increased germinal center formation in the absence of ROR $\gamma$ t. FASEB J. 2016 Feb;30:761-74. DOI: 10.1096/ fj.15-274001

25. Linterman MA, Pierson W, Lee SK, Kallies A, Kawamoto S, Rayner TF, et al. Foxp3+ follicular regulatory $\mathrm{T}$ cells control the germinal center response. Nat. Med. 2011 Jul 24;17: 975-82 DOI: 10.1038/nm.2425

26. Chung Y, Tanaka S, Chu F, Nurieva R, Martinez GJ, Rawal G, et al. Follicular regulatory T cells expressing Foxp3 and Bcl-6 suppress germinal center reactions. Nat. Med. 2011;17: 983-88. DOI: 10.1038/nm.2426

27. Linterman MA, Denton AE. Treg cells and CTLA4: the ball and chain of the germinal center response. Immunity. 2014;41: 876-78. DOI: 10.1016/j.immuni.2014.12.009

28. Dhaeze T, Peelen E, Hombrouck A. Circulating Follicular Regulatory T Cells Are Defective in Multiple Sclerosis. J Immunol. 2015;Aug 1;195(3):832-40. DOI: 10.4049/jimmunol.1500759

29. Quinn JL, Kumar G, Agasing A, Ko RM, Axtell RC. Role of TFH cells in promoting T Helper 17- induced neuroinflammation. Front Immunol. 2018 Feb; 9:382. DOI: $10.3389 /$ fimmu.2018.00382

30. Ireland SJ, Guzman AA, Frohman EM, Monson NL. B cells from relapsing remitting multiple sclerosis patients support neuro-antigen-specific Th17 responses. J Neuroimmunol. 2016 Feb; 291:46-53. DOI: 10.1016/j. jneuroim.2015.11.022

31. Quinn JL, Axtell RC. Emerging role of follicular T Helper cells in multiple sclerosis and experimental autoimmune encephalomyelitis. Int J Mol Sci. 2018 Oct;19(10). pii: E3233. DOI: 10.3390/ijms19103233

32. Pikor NB, Astarita JL, Summers-Deluca L, Ludwin S, Turley SJ, Gommerman L. Integration of Th17and Lymphotoxin-Derived Signals Initiates Meningeal-Resident Stromal Cell Remodeling to Propagate Neuroinflammation. Immunity. 2015 Dec; 43(6):116073. DOI: 10.1016/j.immuni.2015.11.010

33. Balasa R, Maier S, Voidazan S, Hutanu A, Bajko Z,
Motataianu A. An Intricate Mechanism of Action of Avonex in Relapsing Remitting Multiple Sclerosis Patients: Variation of Serum Titre of Interleukin-17A, Interleukin-10 and Transforming Growth Factor- $\beta$. CNS Neurol Disord Drug Targets. 2015;14(6):804-810. DOI: 10.2174/1871527314666150317225441

34. Balasa R, Huţanu A, Bajko Z, Feier C, Pascu I. Does the serum IL-17 titer influence the efficacy of interferon- $\beta$ treatment in multiple sclerosis patients? Rev Romana Med Lab. 2011;19(4):381-9. DOI: $10.1177 / 1352458512468497$

35. Kang Z, Wang C, Zepp J, Wu L, Sun K, Zhao J, et al. Act1 mediates IL-17-induced EAE pathogenesis selectively in NG2+ glial cells. Nat Neurosci. 2013 Oct;16(10):1401-8. DOI: 10.1038/nn.3505

36. Hughes EG, Kang SH, Fukaya M, Bergles DE. Oligodendrocyte progenitors balance growth with self-repulsion to achieve homeostasis in the adult brain. Nat Neurosci. 2013 Jun;16(6): 668-676. DOI: 10.1038/nn.3390

37. Dimou L, Gallo V. NG2-glia and their functions in the central nervous system. Glia 2015 Aug;63(8):1429-51. DOI: $10.1002 /$ glia.22859

38. Zhang CJ, Wang C, Jiang M, Gu C, Xiao J, Chen X, et al. Actl is a negative regulator in T and B cells via direct inhibition of STAT3. Nat Commun. 2018 Jul;9:2745. DOI: $10.1038 / \mathrm{s} 41467-018-04974-3$

39. Nishiyama A, Chang A, Trapp BD. NG2+glial cells: a novel glial cell population in the adult brain. J Neuropathol Exp Neurol. 1999 Nov;58:1113-24. DOI: 10.1097/00005072-199911000-00001

40. Simon C, Gotz M, Dimou L. Progenitors in the adult cerebral cortex: cell cycle properties and regulation by physiological stimuli and injury. Glia. 2011 Jun; 59: 869-81. DOI: $10.1002 /$ glia.21156

41. Domingues HS, Portugal CC, Socodato R, Relvas JB. Oligodendrocyte, astrocyte, and microglia crosstalk in myelin development, damage, and repair. Front Cell Dev Biol. 2016 Jun; 4:71. DOI: 10.3389/ fcell.2016.00071

42. Paintlia MK, Paintlia AS, Singh AK, Singh I. Synergistic activity of interleukin-17 and tumor necrosis factor- $\alpha$ enhances oxidative stress-mediated oligodendrocyte apoptosis. J Neurochem. 2011 Jan;116(4):508-21 DOI: $10.1111 / \mathrm{j} .1471-4159.2010 .07136 . \mathrm{x}$ 